\title{
SurveyWiz and FactorWiz: JavaScript Web pages that make HTML forms for research on the Internet
}

\author{
MICHAEL H. BIRNBAUM \\ California State University, Fullerton, California \\ and Decision Research Center, Fullerton, California
}

\begin{abstract}
SurveyWiz and factorWiz are Web pages that act as wizards to create HTML forms that enable one to collect data via the Web. SurveyWiz allows the user to enter survey questions or personality test items with a mixture of text boxes and scales of radio buttons. One can add demographic questions of age, sex, education, and nationality with the push of a button. FactorWiz creates the HTML for within-subjects, two-factor designs as large as $9 \times 9$, or higher order factorial designs up to 81 cells. The user enters levels of the row and column factors, which can be text, images, or other multimedia. FactorWiz generates the stimulus combinations, randomizes their order, and creates the page. In both programs HTML is displayed in a window, and the user copies it to a text editor to save it. When uploaded to a Web server and supported by a CGI script, the created Web pages allow data to be collected, coded, and saved on the server. These programs are intended to assist researchers and students in quickly creating studies that can be administered via the Web.
\end{abstract}

The Internet can be used as a device to facilitate collection of data in the laboratory, with college students tested by means of Internet-connected computers. The WorldWide Web (Web) can also be used to recruit and test a heterogeneous or specialized sample of participants from around the world. Schmidt (1997a, 2000) and Reips (2000) have reviewed potential advantages and difficulties of such Web-based research and proposed solutions to potential problems.

A number of studies have compared data between laboratory methods and Web-based versions of the same studies, or between people tested by identical methods who were either recruited from the Internet or from the local "subject pool." The trend emerging from such research is that Web studies reach the same conclusions as those conducted in the lab, once the demographics of the participants are taken into consideration (Birnbaum, 1999; Buchanan, 2000; Buchanan \& Smith, 1999; Krantz, Ballard, \& Scher, 1997; Krantz \& Dalal, 2000; Pasveer \& Ellard, 1998; Pettit, 1999; Stanton, 1998). In cognitive psychology, where most research is controlled by computers anyway, it is taken for granted that if an experiment is properly programmed, the same cognitive phenomena will be observed in the lab and on the Web (Francis, Neath, \& Surprenant, 2000; McGraw, Tew, \& Williams, 2000). A survey of "pioneer" Web researchers has found

Thanks are due Jonathan Baron, William Schmidt, and Jeffrey Stanton for their helpful suggestions. This work was supported by National Science Foundation Grant SBR-9410572. Correspondence concerning this article should be addressed to M. H. Birnbaum, Department of Psychology, California State University, P.O. Box 6846, Fullerton, CA 92834-6846 (e-mail: mbirnbaum(a)fullerton.edu). that most considered their Web studies to be successful and plan to conduct future research using this medium (Musch \& Reips, 2000).

On July 1, 1999, 66 projects were linked to the American Psychological Society Web site listing psychological research on the net (Krantz, 1998), approximately double the number linked the previous year. Given the potential to collect large samples of high quality data quickly via the Web, it is reasonable to suppose that this type of research will continue to grow exponentially (Birnbaum, in press).

To collect data by means of the Web, one can upload an HTML form to a Web server, where participants from around the world can use their browsers to read it and respond to its questions. The HTML form can contain various input devices such as text boxes and radio buttons, which allow the participant's responses to be encoded and sent to the investigator by e-mail or to a data file on the server. The participant responds to a text box by typing an answer that can consist of text or a number. Radio buttons require the participant to point and click to select one from several choices.

Table 1 illustrates the technique of HTML forms. The first item accepts the response in a text box 10 characters wide that will accept up to 20 characters. The second item uses a horizontal row of radio buttons, labeled at each end. The "extra" button in the margin is used to code a failure to respond. Its placement also helps the participant to notice that the item has not been completed. A submit button labeled "Done" will cause the action of the form to be executed. In this case, the action sends the data to the investigator by e-mail. Alternately, the action of a Web form can send the collected data to a CGI (common gateway in- 
Table 1

Example of HTML Forms Technique

\begin{tabular}{l}
\hline HTML $>$ \\
$<$ HEAD $>$ TITLE $>$ Example of use of HTML form $<$ TITLE $></$ HEAD $>$ \\
$<$ BODY $>$ \\
$<$ FORM METHOD=post ACTION=-mailto:user@address.domain ENCTYPE="text/plain"> \\
1. How many car accidents have you had? \\
$<$ INPUT TYPE=text NAME=00accidents SIZE=10 MAXLENGTH=20> \\
$<$ P $><$ INPUT TYPE=radio NAME=01 nervous VALUE=" CHECKED $>$ \\
2. I often feel too nervous to drive. $<$ BR $>$ \\
strongly disagree \\
$<$ INPUT TYPE=radio NAME=01 nervous VALUE=1 $>$ \\
$<$ INPUT TYPE=radio NAME=01 nervous VALUE=2> \\
$<$ INPUT TYPE=radio NAME=01 nervous VALUE $=3>$ \\
$<$ INPUT TYPE=radio NAME=01 nervous VALUE=4> \\
$<$ INPUT TYPE=radio NAME=01 nervous VALUE=5> \\
strongly agree $<$ BR $>$ \\
$<$ INPUT TYPE=submit VALUE="Done" $>$ \\
$</$ FORM $>$ \\
$</$ BODY $>$ \\
$</$ HTML $>$ \\
Note-The example above can be tested by typing it into a text editor, saving it as plain ASCII \\
text with an extension of .htm or .html (e.g., test.htm), and loading it in a browser such as \\
Netscape Navigator. Change user@address.domain to your e-mail address, and you will receive \\
the data by e-mail.
\end{tabular}

terface) program that organizes the data and saves them to the server (Schmidt, 1997a).

Writing the HTML for a survey, personality test, or experiment is not difficult once HTML is mastered; however, it can be tedious and time-consuming. It is easy to make errors, creating additional delays. In addition, certain experiments, such as factorial designs composed of text, may require considerable effort to randomize trials and type all of the combinations.

Two Web pages have been developed that act as "wizards" to help researchers, by quickly creating and previewing HTML forms for psychological surveys and within-subjects, factorial experiments. These free Web sites contain JavaScript programs that run on modern browsers, such as Netscape Navigator ( 3 or above) or Internet Explorer ( 4 or above), under any operating system. The research pages can be created and tested on line. The research pages generated by the programs include only HTML, allowing them to run on any browser and platform (even if JavaScript is not enabled).

An explanation of how to use each program is contained in each Web page. Each program is self-contained in a single file that can be viewed by selecting View Source from the browser.

\section{SURVEYWIZ}

SurveyWiz is available from the following URL: http:// psych.fullerton.edu/mbirnbaum/programs/surveyWiz. $\mathrm{htm}$. This program can be used to create simple surveys or personality tests that include questions with a mixture of text boxes and scales of radio buttons. Figure 1 illustrates how to use surveyWiz. Type in a title for the survey and a short name and then press button 1. Start Form. Next, type the first question (e.g., "How many car accidents have you had?") in the space provided under line 2. Then, press button $3 a$. Text Input to create the HTML for a text question. To create a question with a scale of radio buttons, type the question under line 2 and then enter the number of buttons and the labels of the endpoints. The default settings specify a row of 5 buttons, labeled with endpoints of strongly disagree and strongly agree. An example item might be, "I often feel too nervous to drive." Press $3 b$. Radio Button Scale, and the HTML will be added.

The response scale may be the same or different for each radio button item. One can mix questions with radio buttons with questions requesting numerical or text responses. Up to 90 questions can be added. Pushing button 3c. Demographics adds a pre-set list of demographic questions for gender, age, education, and nationality, plus a box for comments. These items can be added at any point in the survey. When all of the questions have been completed, one pushes button 4. Finish the Form. To view the appearance of the new Web page, push button 5. Display. The user can edit the form in the window and press the 5. Display key again to preview the edited form. One can copy and paste the HTML at this point to a text editor, such as NotePad for the PC or SimpleText for the Mac. The Save key does not save, but reminds the user to save in a text editor. Once the HTML has been saved with an extension of $\mathrm{htm}$, the new page can be opened in a 


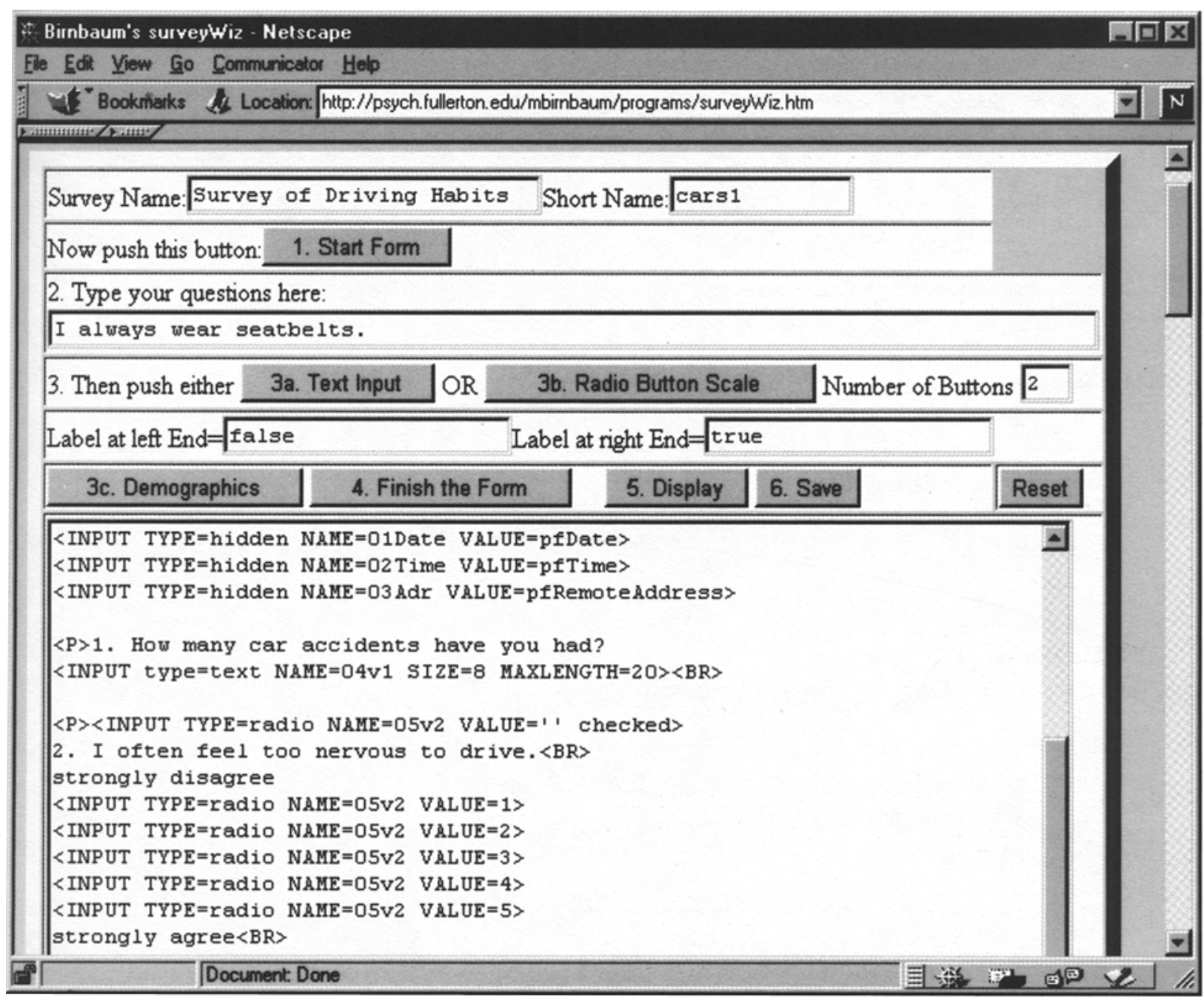

Figure 1. Illustration of the use of surveyWiz. In this example, the investigator is about to enter a true-false item, by pushing button, 3b. Radio Button Scale.

browser. The Reset button erases everything from the form, allowing one to start over. Figure 2 shows how the form, with its text boxes and radio buttons, is displayed in the browser. Using the program is thus as simple as filling in a few boxes with titles, typing the questions, and pushing a few buttons.

\section{FACTORWIZ}

FactorWiz has the URL http://psych.fullerton.edu/ mbirnbaum/programs/factorWiz.htm. The Web page for factorWiz is shown in Figure 3.

The example in Figure 3 helps illustrate what the program does and how to use it. In a classic study, Cliff (1959) found that adverbs multiply the value of adjectives. A person who is very good is judged as better than a person who is good, but a person who is very bad is judged as worse than a person who is $b a d$. The data create a crossover pattern of data that makes a good example for teaching students about interactions. Figure 3 shows how to set up a $4 \times 6$ factorial design of adverbs $\times$ adjectives to (partially) replicate Cliff. The experimenter fills in the experiment's name, Adjectives and Adverbs, which will be printed as the title of the Web page, and a short name, AdjAdv, which will be the first variable in the data. There are four levels of the row factor (adverb) and 6 levels of the column factor (adjectives). The four adverbs are slightly, (no adverb), very, and extremely; the six adjectives are evil, bad, inferior, charming, pleasant, and good. The Separator between factors (lower right of Figure 3) contains a single space that will be placed between the adverb and adjective. Pressing the Make the Form button creates all factorial combinations of row and column levels, randomly reorders the trials, and makes the HTML form, which includes four warmup trials; demographic questions regarding sex, age, education, and nationality; 


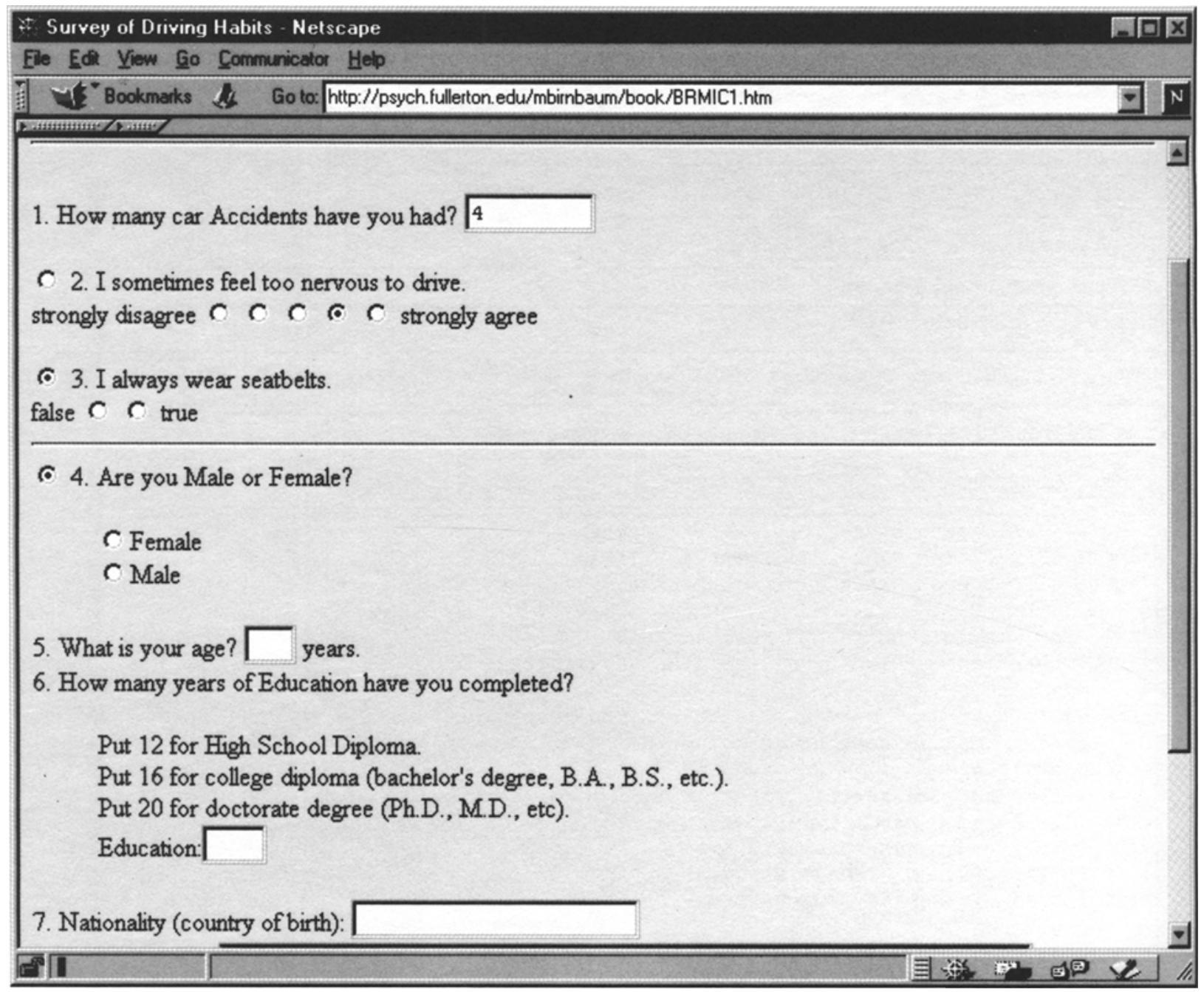

Figure 2. Example of a Web survey made by surveyWiz. This picture illustrates how the page appears to a participant who has answered the first two items.

and a box for comments. The button Make the Form can be pushed several times to create additional random orders, which can be saved in separate files.

The only task remaining is to add the instructions for the task, replacing "(put your instructions here)" with instructions such as the following: "This is a study of impression formation. Your task is to read each description of a person and imagine how much you would like such a person. On each trial, type a number from 1 to 9 to indicate your judgment of liking on a scale from $1=$ dislike very very much to $9=$ like very very much. If you would be neutral (neither like nor dislike the person), type 5." When ready, the HTML displayed in the window is copied into a text editor, from which it is saved with an extension of .htm. The resulting page is displayed in Figure 4. If desired, one can add additional warmup trials, to make sure that all of the stimulus levels have been displayed to the participant before the main experiment.
The row and column factors can themselves be composed of a factorial design with up to 9 cells. For example, the column factor might consist of a $3 \times 3, \mathrm{D} \times \mathrm{E}$, factorial design. The row factor might be a $2 \times 2 \times 2$, $\mathrm{A} \times$ $\mathrm{B} \times \mathrm{C}$, factorial design. The result would be a $2 \times 2 \times$ $2 \times 3 \times 3, \mathrm{~A} \times \mathrm{B} \times \mathrm{C} \times \mathrm{D} \times \mathrm{E}$, factorial design.

Another variation in the use of factorWiz is to use HTML for the row and column factors. This trick allows the construction of Web pages that present images, sound, or other multimedia. For example, an experiment on the Müller-Lyer illusion was created by factorWiz. The stimuli, made in Adobe Pagemaker 5.0, were constructed with a factorial combination of length of line and fin type (angle). They were saved with names such as L3F4.jpg, where the number following the $L$ designated the level of line length and the number following the $F$ represented the fin type (fin angle). The design was a $5 \times 6$, length of line $\times$ fin type, factorial design. The levels of rows and 


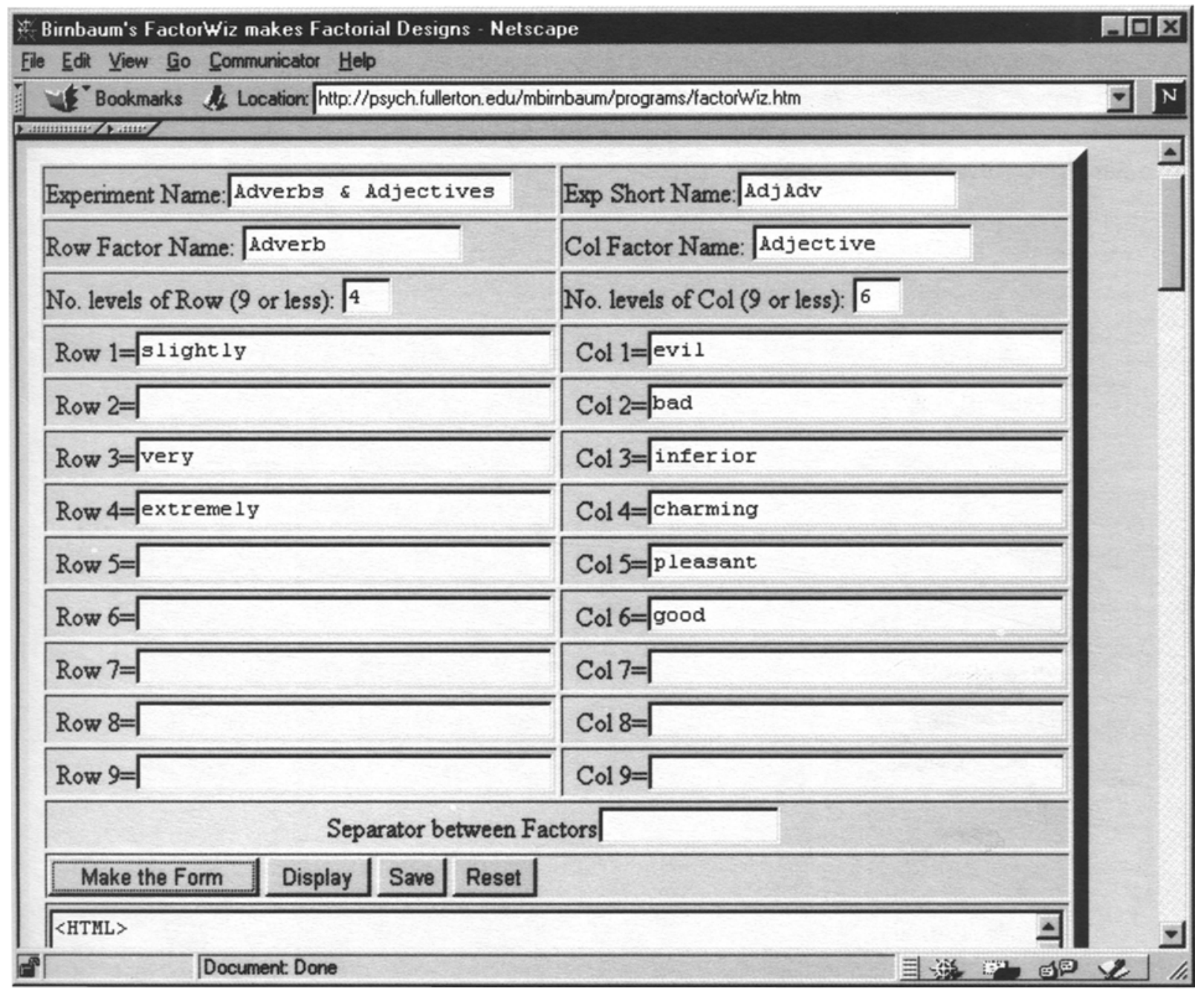

Figure 3. Illustration of the use of factorWiz to investigate how adverbs and adjectives combine. The text area window, below the portion of the window shown, contains the HTML.

columns typed in factorWiz were each a portion of the HTML tag required to display one image. For example, the entry in Row 2 was

$$
<\text { IMG SRC }=\text { L2 }
$$

The entry for Col 5 was as follows:

$$
\text { F5.jpg ALIGN=MIDDLE }><\mathrm{BR}><\mathrm{HR}><\mathrm{BR}>
$$

The Separator was empty, so the combination of Row 2 and $\mathrm{Col} 5$ completed the HTML tags required to display the stimulus of the $(2,5)$ cell-that is, the IMG tag, plus line returns and a horizontal rule to separate the trials:

$$
\begin{aligned}
& <\mathrm{IMG} \text { SRC }=\text { L2F5.jpg ALIGN=MIDDLE }><\mathrm{BR}> \\
& <\mathrm{HR}><\mathrm{BR}>
\end{aligned}
$$

It is important to emphasize that the multimedia stimuli themselves are not made by factorWiz, but that the randomization of trials and HTML to display them can be made by factorWiz. The Müller-Lyer experiment can be viewed at URL http://psych.fullerton.edu/mbirnbaum/ web/MuellerLyer/MLFrame.htm.

Other examples of factorial experiments created with factorWiz include the following:

1. Test of constant weight averaging model of impression formation, replicating Birnbaum (1974, Experiment 1 Replicate 1). How much would you like a person who is sincere and mean? The judge's task was to judge how much he or she would like a person, based on two adjectives, each contributed by a different source, that describe the person (http://psych. fullerton.edu/mbirnbaum/web/ like2.htm).

2. A test of neglect of base rates in Bayesian inference. This study replicated Birnbaum and Mellers (1983), except with the cab problem, which had previously been used to argue for neglect of base rate by Kahneman and Tversky (1973). The judge's task was to estimate the probability that the cab in an accident was blue or green, depending on the proportion of blue cabs in the city and 


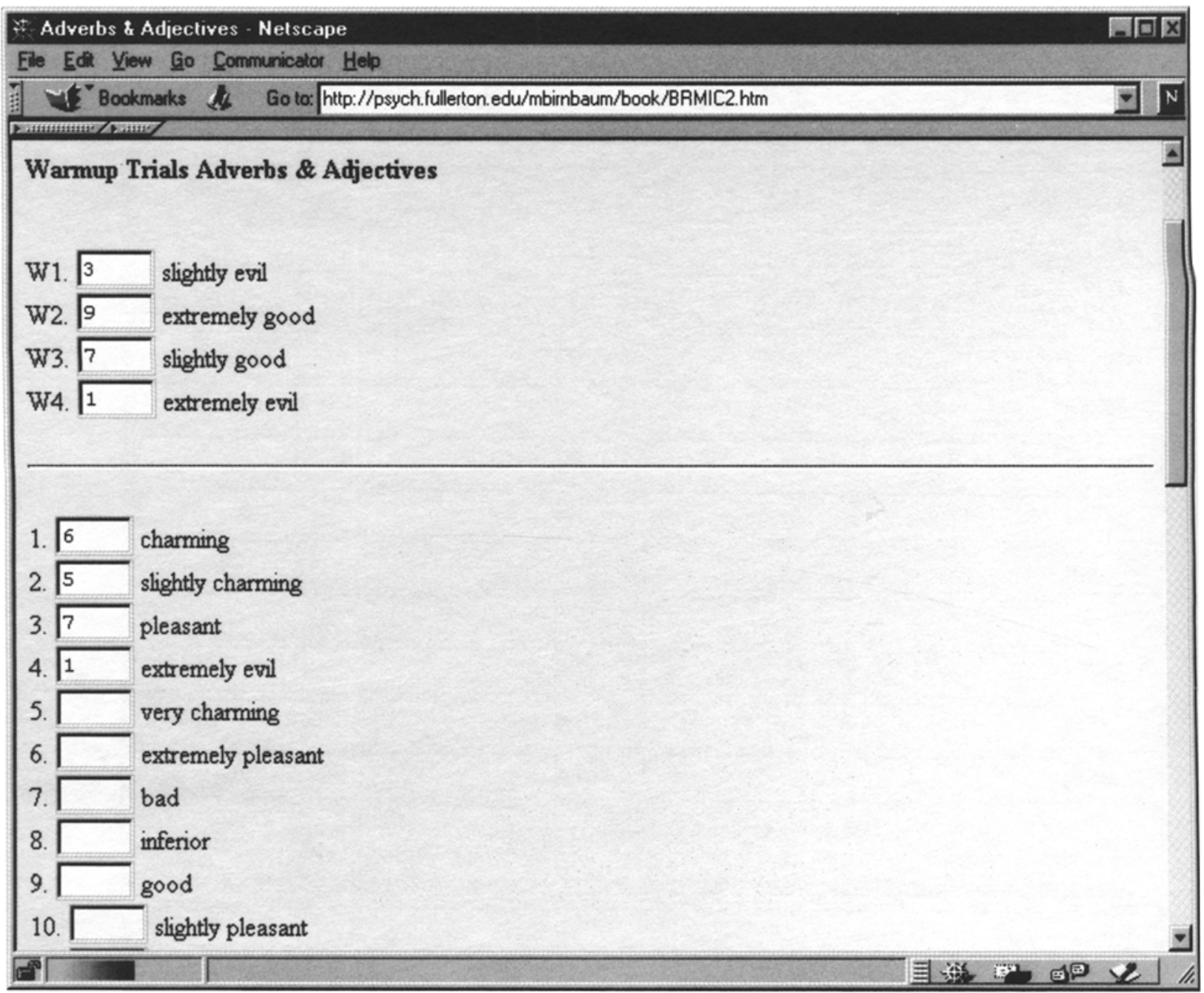

Figure 4. Appearance of Web experiment made by factorWiz, as it would appear to a participant who has completed the first few items.

the testimony of a witness who said that the cab was either "blue" or "green". The credibility of the witness was also varied (http://psych.fullerton.edu/mbirnbaum/web/ bayes.htm).

3. Judged "ratios" and "differences" in prestige of occupations. How much more prestigious is it to be a doctor than to be a factory worker? This study replicated one study by Hardin and Birnbaum (1990) (http://psych. fullerton.edu/mbirnbaum/web/prestige_ratios.htm and http://psych.fullerton.edu/mbirnbaum/web/prestige_diffs htm).

4. Investigation of balance in social judgment. How much would you like John if you love Bill and Bill hates $J o h n$ ? The two independent variables were how much you (the judge) like or dislike Bill and how much Bill likes or dislikes John; the dependent variable was the inference of how much you (the judge) expect to like John (http:// psych.fullerton.edu/mbirnbaum/web/heider.htm).

\section{EDITING THE HTML}

The HTML can be edited in a text editor, in an HTML editor, or directly in the text area window of the surveyWiz or factorWiz Web pages. In both programs, one should replace "(put your instructions here)" with appropriate instructions for the task.

In both programs, the background color of the created page can be altered from the default color by editing BG$\mathrm{COLOR}=$ "color" in the <BODY BGCOLOR="color"> tag. Colors can be represented as color names, such as BGCOLOR="cyan", or as numbers in Red-Green-Blue hexadecimal, such as, BGCOLOR="00fff". In this scheme, $000000=$ black, ffffff $=$ white, ff0000 $=$ red, $00 \mathrm{ff} 00=$ green, and $0000 \mathrm{ff}=$ blue. There are 216 "browsersafe" colors, composed of all $6 \times 6 \times 6$, red $\times$ green $\times$ blue combinations with these six levels of each color: 00 , $33,66,99, \mathrm{cc}$, and ff. Other colors are possible, but they 
may be displayed differently with different browsers and systems.

One can edit the text boxes to increase or decrease their sizes, by editing SIZE $=" 8$ " and MAXLENGTH="20" to other values. To change all of the text boxes, use search and replace in the text editor.

Both programs automatically insert the date, time, and remote address. These variables are supported by the CGI script that is referenced as the default action of the form. Remote address does not identify a person uniquely, but if two submissions arrive at nearly the same time and date with the same remote address, they are probably from the same person (Schmidt, 1997a; Reips, 2000).

When the HTML has been edited, it should be saved with an extension of .htm or .html.

\section{RETRIEVING THE DATA}

The pages created include a default to send the data to a generic CGI protocol which sends the participant to a generic "Thank you" message, and sends the data to a comma separated value (CSV) data file, data.csv, which can be retrieved by FTP by entering the following address in the browser: ftp://guest:guest99@psych.fullerton.edu/ data.csv.

A link in the instructions to each page can be clicked to download the data file, data.csv. Comma separated value (CSV) files can be easily imported to spreadsheet and statistics programs such as Excel and SPSS. In Excel, it is possible to use AutoFilters to filter the data on the first variable, in order to extract only the data from a single experiment (Birnbaum, in press).

The purpose of the generic script is to enable researchers and students to test their pages before they arrange their own scripts. Students and other users of this generic script (and its associated data file) are welcome to use these resources if they agree to allow participants to remain anonymous and to avoid anything sensitive (since data will be stored in a public site). In addition, they should agree not to use others' data without permission, or violate any other ethical principle of the APA code of ethics. Users are advised to download their data frequently, because the file will be cleaned out from time to time on weekends.

One can set ACTION=mailto:user@address.domain, where user@address.domain is the investigator's e-mail address. The appearance of the e-mail can be improved by addition of the ENCTYPE="text/plain" attribute, as in Table 1. When the participant clicks the "submit" button, the data will be sent by e-mail to the address specified. This procedure is useful when one is testing new forms, but it might not be practical for heavy data collection, since it might involve thousands of separate e-mail messages.

The investigator will eventually want to make arrangements to send the data to a script on his or her local server. A script in PERL (together with instructions for installation) that works with surveyWiz and factorWiz can be accessed from both Web pages. PERL is a free program- ming language that is popular for writing CGI scripts (Schwartz, 1998). Once this script is installed on the local server, one can change the URL in the action of the form to the URL of the local script. This PERL script saves data to the local server with a filename matching the short name of the experiment.

The data in the data file created by surveyWiz will be arranged in the order in which they were entered in the surveyWiz. Once they are in HTML, however, the items can be reordered, and the data will still return in the original order (the generic script arranges data in order of the two leading numbers preceding each variable name).

The data in the file created by factorWiz will always be in the factorial order (row 1, col 1), (row 1, col 2), . . (row $1, \operatorname{col} c$ ), (row 2, col 1)... (row $r, \operatorname{col} c$ ), where $r$ and $c$ are the number of rows and columns, which is not the same order as that in which the trials appear in the page. All random orders created by pushing the Make the Form button will return data in the same, factorial order.

For assigning participants to different conditions, such as different random orders of the factorial design, two methods are provided in the factorWiz and surveyWiz Web pages. One method is to use a server-side script in PERL. Another method is to use another Web page, with a JavaScript routine to assign participants to conditions on the basis of a random number. If JavaScript is not installed, the participant is asked to click his or her birth month, and assignment is based on birth month. Both of these techniques can be reached by links in the Web pages.

\section{UPLOAD TO THE WEB SERVER}

When the form has been thoroughly tested, it can be placed in an appropriate location on a Web server. Before a survey or experiment is posted to the Web, however, one should practice not only the usual precautions (ethics review, testing oneself with the protocol, testing the scheme for data analysis, etc.), but also exercise even greater than usual care. One should strive to ensure that the research materials will not be offensive to people of cultures outside of the investigator's usual experience, that the research is well thought out, and that the study will answer a scientific question. There is great potential on the Internet to waste the precious time of a large number of participants who are kind enough to assist us with research. Use of deception or poorly conceived research would give psychology a bad reputation and discourage those who might otherwise participate in on-line research.

\section{COMPARISON WITH OTHER METHODS}

Each of the experiments listed above gave clean data that replicated findings that have been well established by laboratory methods (Birnbaum, in press). The comparison of time and effort needed to conduct the lab and Web versions of the same studies is impressive. When the first experiment of Birnbaum (1974) was done, in 1968, the labor of randomizing trials, typing the stimuli, and re- 
producing paper copies required a week. Data were collected in the lab over many weeks. Data sheets were recoded by hand from random to factorial order by means of printed coding matrices that were prepared for each random order; finally, the data were keypunched and verified on cards. Data recoding, keypunching, and checking required about 10-12 min per participant. The Web method computerizes stimulus randomization, data entry, and recoding stages, thus cutting the time and effort required by a factor of 10 . What used to take 6 months can now be done in a few weeks.

A number of products can do what surveyWiz does. WWW Survey Assistant (SA) is probably the best of these (Schmidt, 1997b). Noncommercial educational users can use SA without cost, and it is superior to comparable commercial products because it supports parallel construction of HTML and CGI scripts to process and filter the data. See http://or.psychology.dal.ca/ wcs/hidden/ SAdocs/competition.html.

SA was designed for people who do not know programming or HTML, and it can handle a greater variety of input formats than surveyWiz can. Although surveyWiz is more restricted than SA, surveyWiz is easier to learn and use for a person who knows a little HTML. The author is unaware of software besides factorWiz, free or otherwise, that creates and randomizes factorial designs, as factorWiz does.

\section{REFERENCES}

BiRNBAUM, M. H. (1974). The nonadditivity of personality impressions. Journal of Experimental Psychology, 102, 543-561.

BirnbaUm, M. H. (1999). Testing critical properties of decision making on the Internet. Psychological Science, 10, 399-407.

BIRNBAUM, M. H. (in press). Introduction to behavioral research on the Internet. Upper Saddle River, NJ: Prentice Hall.

Birnbaum, M. H., \& Mellers, B. A. (1983). Bayesian inference: Combining base rates with opinions of sources who vary in credibility. Journal of Personality \& Social Psychology, 45, 792-804.

Buchanan, T. (2000). Potential of the Internet for personality research. In M. H. Birnbaum (Ed.), Psychological experiments on the Internet (pp. 121-140). San Diego, CA: Academic Press.

Buchanan, T., \& SMith, J. L. (1999). Using the Internet for psychological research: Personality testing on the World-Wide Web. British Journal of Psychology, 90, 125-144.

ClifF, N. (1959). Adverbs as multipliers. Psychological Review, 66, 27 44.
Francis, G., Neath, I., \& Surprenant, A. M. (2000). The cognitive psychology online laboratory. In M. H. Birnbaum (Ed.), Psychological experiments on the Internet (pp. 267-283). San Diego, CA: Academic Press.

Hardin, C., \& Birnbaum, M. H. (1990). Malleability of "ratio" judgments of occupational prestige. American Journal of Psychology, $103,1-20$.

KAHNEMAN, D., \& TVERSKY, A. (1973). On the psychology of prediction. Psychological Review, 80, 237-251.

KRANTZ, J. H. (1998). American Psychological Society-Psychological Research on the Net. Retrieved July 1, 1999 at http://psych.hanover.edu/ APS/exponnet.html.

Krantz, J. H., Ballard, J., \& SCher, J. (1997). Comparing the results of laboratory and World-Wide Web samples on the determinants of female attractiveness. Behavior Research Methods, Instruments, \& Computers, 29, 264-269.

KrantZ, J. H., \& Dalal, R. (2000). Validity of Web-based psychological research. In M. H. Birnbaum (Ed.), Psychological experiments on the Internet (pp. 35-60). San Diego: Academic Press.

McGraw, K. O., Tew, M. D., \& Williams, J. E. (2000). PsychExps: An On-Line Psychology Laboratory. In M. H. Birnbaum (Ed.), Psychological experiments on the Internet (pp. 219-233). San Diego, CA: Academic Press.

MusCh, J., \& Reips, U.-D. (2000). A brief history of Web experimenting. In M. H. Birnbaum (Ed.), Psychological experiments on the Internet (pp. 61-87). San Diego, CA: Academic Press.

Pasveer, K. A., \& ElLARD, J. H. (1998). The making of a personality inventory: Help from the WWW. Behavior Research Methods, Instruments, \& Computers, 30, 309-313.

Petrit, F. A. (1999). Exploring the use of the World Wide Web as a psychology data collection tool. Computers in Human Behavior, 15, $67-71$.

REIPS, U.-D. (2000). The Web experiment method: Advantages, disadvantages, and solutions. In M. H. Birnbaum (Ed.), Psychological experiments on the Internet (pp. 89-117). San Diego, CA: Academic Press.

SCHмIDT, W. C. (1997a). World-Wide Web survey research: Benefits, potential problems, and solutions. Behavior Research Methods, Instruments, \& Computers, 29, 274-279.

SCHMIDT, W. C. (1997b). World-Wide Web survey research made easy with WWW Survey Assistant. Behavior Research Methods, Insiruments, \& Computers, 29, 303-304.

SCHMidT, W. C. (2000). The server-side of psychology Web experiments. In M. H. Birnbaum (Ed.), Psychological experiments on the Internet (pp. 285-310). San Diego, CA: Academic Press.

SCHWARTZ, A. (1998). Tutorial: PERL, a psychologically efficient reformatting language. Behavior Research Methods, Instruments, \& Computers, 30, 605-609.

Stanton, J. M. (1998). An empirical assessment of data collection using the Internet. Personnel Psychology, 51, 709-725.

(Manuscript received December 18, 1998; revision accepted for publication November 20, 1999.) 